\title{
Efeito da ansiedade, racionalidade e uso de recursos financeiros na relação entre materialismo e o comportamento de compra impulsivo
}

\author{
Effect of anxiety, rationality and use of financial resources on the relationship \\ between materialism and impulsive buying behavior
}

\author{
Fabio Machado-da-Silva ${ }^{1}$ \\ Adriano Maniçoba da Silva ${ }^{2}$ \\ Rossimar Laura Oliveira ${ }^{3}$ \\ Clayton de Oliveira Pires ${ }^{4}$
}

\begin{abstract}
Resumo
O processo de decisão de compra envolve fatores racionais e emocionais, os quais podem definir como os consumidores reagem aos estímulos de marketing. O objetivo deste estudo foi avaliar o efeito do materialismo sobre a impulsividade nas compras, tendo "ansiedade", "racionalidade" e "uso de recursos financeiros disponíveis" como variáveis mediadoras. Uma surveyon-linecom 244 indivíduos foi aplicada e os dados foram analisados por meio de modelagem de equações estruturais. Os resultados indicaram que o materialismo teve influência indireta sobre a impulsividade nas compras, sendo sua relação mediada por ansiedade, racionalidade e uso de recursos financeiros. Os resultados também indicaram que são consumidores mais impulsivos os seguintes grupos: (1) as mulheres, (2) os que mais utilizam limite do cartão de crédito, (3) os que optam por maior prazo de pagamento e (4) os que têm maior dispêndio do que seus rendimentos suportam. $\mathrm{O}$ estudo contribui para melhorar o entendimento sobre o comportamento do consumidor quanto a construtos que podem afetar a impulsividade, podendo sugerir formas eficazes de atingir o mercado-alvo.
\end{abstract}

Palavras-chave: Materialismo. Impulsividade nas Compras. Ansiedade. Racionalidade. Uso de Recursos Financeiros.

\begin{abstract}
The purchasing decision process involves rational and emotional factors, which can define how consumers react to marketing stimuli. The objective of this paper was to evaluate the effect of Materialism on Impulsive buying behavior, and Anxiety, Rationality and Use of Financial Resources as mediating variables. The online survey of 244 individuals was obtained and the data were analyzed using structural equation modeling. The results indicated that materialism had indirect effect on impulsive buying behavior, and their relation was mediated by anxiety, rationality and use of financial resources. The results also indicated that the groups: (1) women, (2) those who use the credit card limit, (3) those who opt for longer payment terms and (4) those who spend more than their income support are more impulsive consumers. Understanding consumer behavior from constructs that can affect impulsive buying behavior may result in more effective ways to reach the target market.
\end{abstract}

Keywords: Materialism. Impulsive buying behavior. Anxiety. Rationality. Use of Financial Resources.

\section{Introdução}

A concorrência tem pressionado os gestores a desenvolverem estratégias que objetivam a conquista de vantagem competitiva, especialmente com ações que consideram os tipos de comportamentos que cada

Doutor em Administração - FGV-SP e Professor no Instituto Federal de São Paulo. Brasil. Afiliação: InstitutoFederal de São Paulo. Lattes: http:// lattes.cnpq.br/3344019944224399 Email: fabiomachado@ifsp.edu.br

2 Doutor em Administração - Universidade de São Paulo e Professor no Instituto Federal de São Paulo. Brasil. Afiliação: Universidade de São Paulo. Lattes: http://lattes.cnpq.br/6644799917139100 Email: adrianoms@ifsp.edu.br

3 Doutoranda em Administração Universidade de São Paulo e Professora no Instituto Federal de São Paulo. Brasil. Afiliação: Instituto Federal de São Paulo. Lattes: http://lattes.cnpq.br/7857384676441084 Email: rossimar.laura@ifsp.edu.br

4 Professor no Instituto Federal de São Paulo. Brasil. Afiliação: InstitutoFederal de São Paulo. Lattes: http://lattes.cnpq.br/9831134856008226 Email: clayton.pires@ifsp.edu.br 
grupo de consumidores apresenta, permitindo uma abordagem customizada e voltada às características do público-alvo.

Os apelos de marketing devem ser precedidos por estudos minuciosos acerca das características dos consumidores, entre as quais está o nível de impulsividade nas compras. A compra impulsiva é espontânea e sem reflexão, estimulada pelo contexto e pela possibilidade de aquisição imediata, que domina o cliente por meio de atração emocional (DIAS,2014).A compra impulsiva está ligada à importância atribuída à aquisição de bens materiais e à busca por satisfação imediata, sendo uma ferramenta de busca pela felicidade (DITTMAR, 2005; GOLDSMITH;CLARCK,2012).

A impulsividade é uma característica de personalidade presente em pessoas que tomam decisões de compra com pouca reflexão e por razões emocionais (YOUN; FABER, 2000), mesmo sendo comuns as emoções negativas após a compra impulsiva e o medo das dívidas (PORPINO; PARENTE, 2014).

Apesar dos determinantes da compra impulsiva já terem sido objeto de diversas pesquisas (BADGAIYAN; VERMA, 2014), não se encontrou estudos anteriores que avaliaram a relação entre materialismo e impulsividade quando mediada por outros construtos relevantes. Dessa forma, buscase responder à seguinte questão: Qual o efeito da ansiedade, da racionalidade e do uso de recursos financeiros disponíveis na relação entre materialismo e o comportamento de compra impulsivo?

Portanto, o objetivo deste estudo foi avaliar o efeito do materialismo sobre a impulsividade nas compras, tendo "ansiedade", "racionalidade" e "uso de recursos financeiros disponíveis" como variáveis mediadoras. O procedimento surveyfoi aplicado a 244 indivíduos e os dados foram analisados por meio de equações estruturais. Os resultados mostram que, se um indivíduo for ansioso, usar seus recursos financeiros e for mais emocional que racional, tenderá a ser impulsivo.

Este trabalho contribui para entender o papel das variáveis mediadoras no impacto do materialismo no comportamento impulsivo,podendo ser útil para que gestores avaliem quais variáveis potencializam o comportamento impulsivo de consumidores materialistas e elaborem estratégias para determinadas características do público-alvo. O uso de estratégias de marketing por meio de recursos promocionais, visuais e textuais pode ser relevante para o alcance desses objetivos. Alguns exemplos de ações são as ofertas-relâmpago, que despertam a ansiedade com o uso de apelos emocionais, ou o uso de ações que reforcem o status e a sensação de poder dos consumidores materialistas(CUNHA,2012).

\section{Modelo de pesquisa e hipóteses}

O fenômeno do comportamento de compra impulsivo pode ser considerado para condições além das compras realizadas de maneira inesperada(STEFANINI; OLIVEIRA, 2014). Os estudos nessa área começaram na década de 1980. Em trabalho seminal, Rook (1987) define a compra impulsiva como aquela que não é planejada, em situação em que estímulos podem afetar o consumidor e fazê-lo decidir pela compra.Em complemento a esse conceito, Piron (1991) considera a falta de planejamento na compra, a existência de estímulo externo e a decisão de compra realizada no local como os principais determinantes da compra impulsiva. O autor destaca como principal explicação para o fenômeno o comportamento emotivo do consumidor.

Nessa linha de raciocínio, a compra impulsiva é aquela que não foi planejada, na qual a ocasião e a presença física do objeto exercem influência (TEIXEIRA; HERNADEZ,2012). Nesse momento, o consumidor é tomado por um desejo repentino e urgente, podendo resultar na sensação temporária de perda de controle e ausência de preocupação com as consequências (BLACKWELL; MINIARD; ENGEL, 2008; COSTA; LARÁN, 2006).

Diversos instrumentos têm sido utilizados para mensurar o comportamento impulsivo de compra e retratam, entre outros aspectos, a espontaneidade (MICKS, 1996; ROOK; FISHER, 1995; WEUN et al., 1997), a racionalidade, o planejamento (DONTHU; GILLILANDS, 1996; MICK'S, 1996; PURI'S, 1996; ROOK; FISHER, 1995; WEUN et al., 1997), os benefícios hedônicos (PURI'S, 1996; ROOK; FISHER, 1995; WEUN et al., 1997), o estado emotivo (PURI'S, 1996; ROOK; FISHER, 1995), o materialismo (GOLDSMITH; CLARCK,2012; XIAO; NICHOLSON, 2013) e as influências externas (PURI'S, 1996). 
Rook e Fisher (1995) elaboraram um dos instrumentos mais utilizados para mensuração da impulsividade nas compras. Esses autores reforçam a ideia da compra sem reflexão e espontânea, em que o consumidor é atraído emocionalmente pelo objeto físico que está diante dele, visando gratificação imediata. Da mesma forma, para Arnold, Price e Zinkhan (2004), a impulsividade está relacionada à vontade repentina e irresistível de realizar a compra imediatamente.

A percepção de risco está negativamente relacionada à compra sem reflexão (MISHRA et al., 2014). Essa percepção pode ser interpretada como a ponderação racional dos próprios desejos e necessidades, chamada de racionalidade. Define-se racionalidade como um traço psicológico pessoal em que se privilegia, antes da tomada de uma decisão, a razão. Já a emotividade é o estado no qual as pessoas privilegiam seus sentimentos e emoções antes da tomada de decisão (ASSMAR; RODRIGUES, 1989). Um indivíduo racional nas compras dificilmente deixará aspectos emotivos influenciarem demasiadamente o processo de aquisição de um novo produto ou serviço. Da mesma forma, um consumidor extremamente emotivo terá mais dificuldades para conduzir reflexões lógicas acerca da compra (MISHRA et al., 2014).

A compra impulsiva está intimamente ligada a um sistema de valores materialistas que guiam os indivíduos a utilizarem a compra como ferramenta para atingir a felicidade ou satisfação sobre a vida de maneira imediatista, atribuindo um significado simbólico a esse ato,que está acompanhado por maior bemestar pessoal (DITTMAR,2005; GOLDSMITH;CLARCK,2012).

Richins e Dawson (1992) definem o materialismo como a importância atribuída à posse e à aquisição de bens materiais no alcance de objetivos de vida ou estados desejados. Contudo, a relação do materialismo com alguns indicadores, como felicidade, satisfação de vida, saúde mental e saúde psíquica, é negativa.

Segundo Youn e Faber (2000), a impulsividade é uma característica de personalidade de grande importância no âmbito do comportamento do consumidor. Em sua pesquisa, os autores caracterizam indivíduos impulsivos como pessoas que agem espontaneamente, de forma imprudente e negligente, que tomam decisões com pouca reflexão e cujas nuances emocionais são bastante visíveis. Somando a um perfil materialista, é provável que o indivíduo gaste os recursos financeiros disponíveis com mais facilidade, deixando de poupar e de realizar planejamento financeiro de médio e longo prazo.Porpino e Parente (2014) apontam que são comuns emoções negativas pós-compra impulsiva e esses indivíduos apresentam um grande medo do endividamento.

Assumindo que fatores situacionais podem ter influência positiva sobre a compra por impulso (AMOS et al., 2014), e que materialismo, em estudos anteriores, apresentou relação positiva com o comportamento de compra impulsivo (BADGAIYAN; VERMA, 2014), pode-se definir a primeira hipótese:(H1)O "materialismo" está positivamente associado ao "uso de recursos financeiros disponíveis".

Sendo o materialismo manifestado algumas vezes pelo desejo de posse imediata, levando o indivíduo à compra impulsiva (DITTMAR, 2005), assume-se que tal indivíduo tenha menor nível de racionalidade. Desta relação pode-se enunciar a segunda hipótese:(H2) O "materialismo" está negativamente associado à "racionalidade".

Há evidências de que indivíduos materialistas julgam o seu próprio sucesso e o dos outros pelo número de bens, o que gera ansiedade pela compra de novos itens e frustração quando há comparação com indivíduos de maior posse (RICHINS; DAWSON,1992). Ansiedade é um importante determinante da intenção comportamental na teoria social cognitiva (YANG; FORNEY, 2013) e diz respeito à vigilância em relação à rejeição e ao abandono (FRALEY et al., 2000; VLACHOS, 2010), também pela tensão, preocupação e antecipação de possíveis cenários futuros negativos (CRASKE, 2009). A ansiedade se relaciona com o consumo, pois pessoas muito ansiosas desejam emoções positivas nas suas relações comerciais para compensar as emoções negativas e carências que vivem no âmbito pessoal (FELIPE et al., 2017; VLACHOS, 2010).Assim, pode-se elencar a terceira hipótese:(H3)O “materialismo" está positivamente associado à "ansiedade".

De acordo com estudos anteriores que apontam o materialismo como determinante do comportamento de compra impulsivo (BADGAIYAN; VERMA, 2014; PODOSHEN; ANDRZEJEWSKI, 2012), pode-se definir a seguinte hipótese:(H4)O "materialismo" está positivamente associado à "impulsividade nas compras". 
O uso da disponibilidade de recursos financeiros deve ser considerado no estudo da impulsividade nas compras (GOLDSMITH;CLARCK,2012). Para Amos et al. (2014), o uso exagerado de recursos financeiros disponíveis faz parte dos chamados fatores situacionais, que podem levar um indivíduo ao comportamento impulsivo. Nesse contexto, um indivíduo que gasta exageradamente seus recursos financeiros disponíveis tende a ser menos racional nas compras. Sendo assim, pode-se enunciar a seguinte hipótese: (H5)O "uso de recursos financeiros disponíveis" está negativamente associado à "racionalidade".

Indivíduos que tenham maior dispêndio de recursos financeiros, em comparação, excetuando-se casos em que tenham níveis menores de renda, podem ter inviabilizado seu planejamento financeiro, de modo a se comportarem de forma mais impulsiva nas compras (BEATTY, FERRELL, 1998; VELUDODE-OLIVEIRA; IKEDA; SANTOS,2004). Ainda que o consumidor tenha nível menor de renda, a sua capacidade racional de planejamento faria com que ele conseguisse evitar gastos desnecessários e, consequentemente, evitar uso da totalidade de seus rendimentos (AMOS et al., 2014). Sendo assim, a sexta hipótese pode ser enunciada da seguinte forma:(H6)O "uso de recursos financeiros disponíveis" está positivamente associado à "impulsividade nas compras".

A partir da relação positiva entre ansiedade e comportamento de compra impulsivo (RICHINS; DAWSON,1992), e da relação negativa entre racionalidade e o mesmo comportamento (GILLILANDS, 1996; PURI'S, 1996) espera-se, tal como apresentado na sétima hipótese, a seguinte hipótese: (H7)A "ansiedade" está negativamente associada à "racionalidade".

Tal como já explorado, é esperada relação positiva entre impulsividade e ansiedade (RICHINS; DAWSON,1992), o que pode ser enunciado na oitava hipótese:

(H8)A “ansiedade" está positivamente associada à "impulsividade nas compras".

Por fim, a partir dos argumentos utilizados, a racionalidade terá influência negativa no comportamento de compra impulsivo (ROOK; FISHER, 1995), o que será testado por meio da nona hipótese:

(H9)A "racionalidade" está negativamente associada à "impulsividade nas compras".

Ao dispor todas as relações enunciadas nas hipóteses na forma de um modelo de pesquisa, pôde-se apresentar as relações esboçadas na figura 1.

Figura 1: Modelo de Pesquisa e Hipóteses

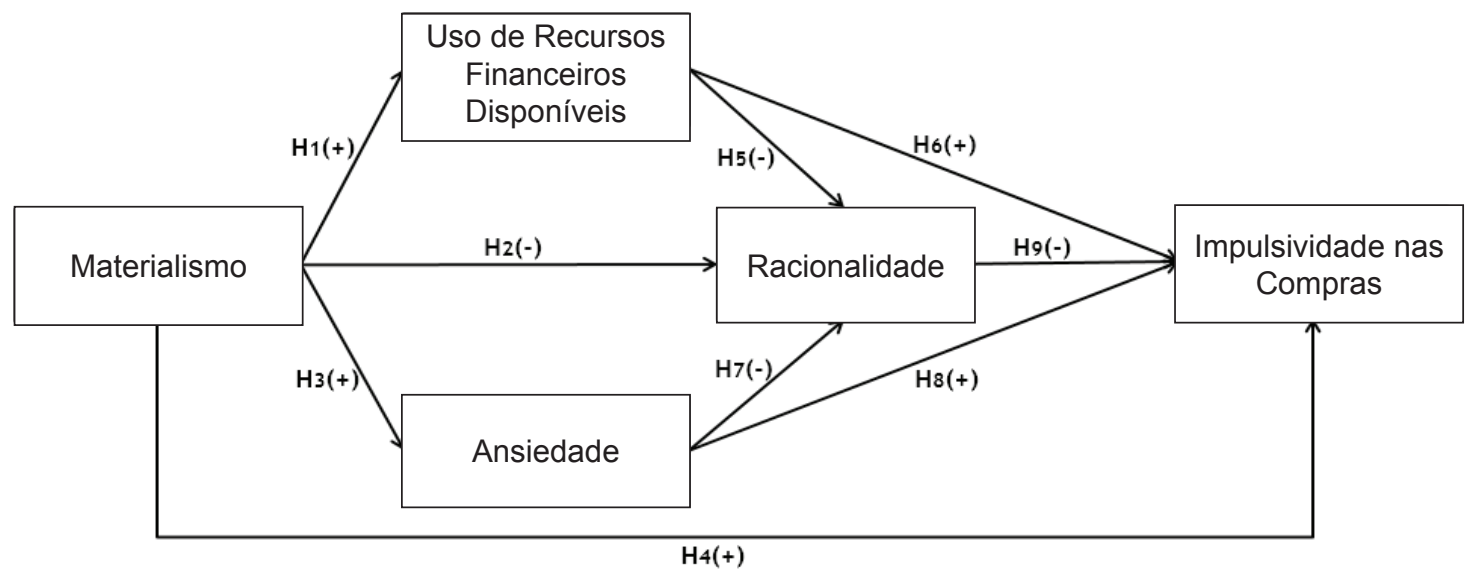

Fonte: Elaborado pelos autores(2015).

\section{Metodologia}

Este estudo, de natureza aplicada e com característica de pesquisa explicativa, utilizou o procedimento tipo surveyon-linecom 244 indivíduos residentes no estado de São Paulo, com outliers verificados. Para identificar o tamanho da amostra mínima necessária,foi utilizado o software Gpower 3.1. Conforme orientações de Faul et al. (2009), foi identificada a variável latente que recebe maior número de setas, a qual, no caso deste estudo, foi o construto "impulsividade nas compras", com quatro variáveis 
independentes ligadas a ele. Considerando os parâmetros tamanho do efeito $\mathrm{f}^{2}$ igual a 0.15 e poder do teste igual a 0.999, conforme indicado em Cohen (1988) e Hair et al. (2014), o software apresenta um F crítico igual a 2.2519 e recomenda amostra mínima com 244 indivíduos. Trata-se de uma amostra por conveniência obtida a partir da distribuição do questionário via e-mail e redes sociais.

Os construtos "racionalidade", "materialismo", "uso de recursos financeiros disponíveis" e "impulsividade nas compras" foram adaptados de Puri(1996), Xião e Nicholson (2013), Amos et al. (2014) e Rook e Fisher (1995), respectivamente. O construto "ansiedade" foi desenvolvido pelos autores e seu conteúdo foi validado por dois especialistas da área de Psicologia do consumo. Para averiguar possíveis problemas de escala e de conteúdo, um pré-teste foi aplicado com 98 indivíduos, sendo o instrumento final composto por 17 questões para identificar características demográficas, geográficas e de padrão de consumo, além de 37 questões dispostas em escala Likert de cinco pontos para mensurar as variáveis do modelo.

O estudo aplicou Modelagem de Equações Estruturais - MEE em uma abordagem confirmatória. O uso de SEM é indicado quando se deseja examinar uma série de relações de dependência de forma simultânea (HAIR et al., 2009). O modelo foi estimado por PLS-PM (PartialLeast Square Path Modeling), considerada uma técnica vantajosa quando se possui um grande número de parâmetros a estimar (CHIN; NEWSTED, 1999).

A variável dependente "impulsividade" foi composta por três questões, sendo uma delas o cenário de decisão, adaptado de Rook e Fisher (1995): "Imagine-se como um(a) estudante universitário(a) de 21 anos de idade que trabalha meio período. Quatro dias antes de receber seu próximo salário, há apenas $R \$ 80,00$ de saldo em sua conta. Você precisa comprar uma blusa comum de frio para usar durante uma visita ao ar livre organizada pela universidade daqui a dois dias. Após o trabalho, você vai ao shopping para comprar a blusa que custa $R \$ 59,90$. Andando pelos corredores, encontra um excelente sapato à venda por apenas $R \$ 79,90$ durante a queima de estoque de uma loja que você gosta muito". O respondente poderia escolher entre uma das alternativas: (a) Mesmo gostando da blusa, decidiria não comprar porque tenho pouco dinheiro; (b) Eu compraria apenas a blusa e não olharia mais para o sapato; (c) Eu compraria a blusa após ter "namorado" muito o sapato, sem comprá-lo; (d) Eu compraria a blusa e o sapato com cartão de crédito; (e) Eu compraria a blusa, o sapato, além de um cinto que combine com o sapato, também com cartão de crédito.

As outras duas questões utilizadas para compor a variável dependente foram a impulsividade declarada, que variou de 1 a 5 , e a impulsividade em duas situações de compra, uma relacionada à compra de produtos de consumo e outra relacionada à compra de vestuário. Foi computada a média das questões de modo que a variável dependente se tornasse contínua para a análise de equações estruturais. Para a realização de testes adicionais em relação a outras variáveis de perfil, a variável dependente foi transformada em variável dicotômica. Para tanto, foi utilizado como ponto de corte o valor 2,75 , sendo que observações iguais ou maiores que essesvalores foram classificados como impulsiva e, quando abaixo desse valor, como não impulsiva.

\section{Análise dos Resultados}

As tabelas 1 e 2 apresentam a amostra do estudo e a análise de dependência entre variáveis categóricas, respectivamente. 
Tabela 1 - Caracterização da amostra $(n=244)$

\begin{tabular}{|c|c|}
\hline Variável & Distribuição (em percentual \%) \\
\hline Sexo & Masculino: $33,20 \%$ |Feminino: $66,80 \%$ \\
\hline Idade & $\begin{array}{l}\text { Até } 20 \text { anos: } 15,57 \% \text { |de } 21 \text { a } 30 \text { anos: } 38,52 \% \text { |de } 31 \text { a } 40 \text { anos: } 29,92 \% \\
\text { |mais de } 40 \text { anos: } 15,98 \%\end{array}$ \\
\hline Estado civil & Solteiro(a): $54,10 \%$ | Casado(a): $37,30 \%$ | Outro: $8,61 \%$ \\
\hline Escolaridade & $\begin{array}{l}\text { Até Nível Médio: } 51,23 \% \text { | Graduação: } 21,31 \% \text { | Especialização: } 17,62 \% \text { | } \\
\text { Mestrado/Doutorado: 9,84\% }\end{array}$ \\
\hline Renda & $\begin{array}{l}\text { Sem Renda: } 10,66 \% \text { | Até } 1 \text { Salário: } 9,43 \% \text { | Até } 3 \text { Salários: } 37,30 \% \text { | Até } 6 \\
\text { Salários: } 25,82 \% \text { | Mais de } 6 \text { Salários: } 16,80 \%\end{array}$ \\
\hline Responsável financeiro da família & Sim: 36,48\% |Não: 63,52\% \\
\hline Número de dependentes & $\begin{array}{l}\text { Sem dependentes: } 52,87 \% \text { | } 1 \text { ou } 2 \text { dependentes: } 36,48 \% \text { | } 3 \text { ou mais } \\
\text { dependentes: } 10,66 \%\end{array}$ \\
\hline Número de cartões de crédito & $\begin{array}{l}\text { Não possui cartão: } 27,87 \% \mid 1 \text { cartão: } 38,52 \% \text { | } 2 \text { cartões: } 20,08 \% \text { | mais de } \\
2 \text { cartões: } 13,52 \%\end{array}$ \\
\hline Uso do limite de cartão & $\begin{array}{l}\text { Não usa cartões: } 27,87 \% \text { |Até } 20 \% \text { do limite: } 30,33 \% \text { |Até } 50 \% \text { do limite: } \\
24,59 \% \text { |Mais de } 50 \% \text { do limite: } 17,21 \%\end{array}$ \\
\hline $\begin{array}{l}\text { Preferência por parcelamento } \\
\text { (produtos de até } r \$ 500,00 \text { ) }\end{array}$ & $\begin{array}{l}\text { À Vista: } 34,84 \% \text { | Até } 3 \text { vezes: } 35,66 \% \text { | Até } 6 \text { vezes: } 17,21 \% \text { | Mais de } 6 \\
\text { vezes: } 12,30 \%\end{array}$ \\
\hline $\begin{array}{l}\text { Prazo para pagamento (Produtos } \\
\text { de até } r \$ 2000,00 \text { ) }\end{array}$ & $\begin{array}{l}\text { À Vista: } 12,70 \% \text { | Até } 3 \text { vezes: } 13,52 \% \text { | Até } 6 \text { vezes: } 16,39 \% \text { | Mais de } 6 \\
\text { vezes: } 57,38 \%\end{array}$ \\
\hline Perfil de poupador & $\begin{array}{l}\text { Não poupa: } 48,36 \% \text { |Poupa até } 10 \% \text { do salário: } 27,05 \% \text { | Poupa até } 30 \% \\
\text { do salário: } 15,16 \% \text { |Poupa até } 50 \% \text { do salário: } 6,56 \% \text { | Poupa mais de } 50 \% \\
\text { do salário: } 2,87 \%\end{array}$ \\
\hline Perfil de gastador & $\begin{array}{l}\text { Não gasta mais do que sua remuneração: } 63,11 \% \text { |Gasta até } 10 \% \text { além do } \\
\text { salário: } 19,26 \% \text { | Gasta até } 30 \% \text { além do salário: } 8,61 \% \text { | Gasta até } 50 \% \\
\text { além do salário: } 3,28 \% \text { | Gasta mais de } 50 \% \text { além do salário: } 5,74 \%\end{array}$ \\
\hline
\end{tabular}

Fonte: Elaborado pelos autores(2015).

As demais variáveis categóricas (idade; estado civil; escolaridade; renda; número de dependentes; número de cartões de crédito; prazo para pagamento -produtos de até $\mathrm{R} \$ 2000,00$; e perfil de poupador) não mostraram significância nos testes de dependência com a variável binária dependente. Outros testes (Qui-quadrado) foram processados para avaliar relações de dependência. Os resultados podem ser conferidos na tabela 3.

Tabela 2 - Dependência entre variáveis categóricas e a variável binária dependente (impulsivo; não impulsivo) - Teste qui-quadrado

\begin{tabular}{|l|c|c|l|}
\hline Variável categórica & Qui-quadrado & Valor-p & Conclusão \\
\hline Sexo & 14,633 & 0,000 & $\begin{array}{l}\text { Mulheres declararam serem mais impulsivas do que os } \\
\text { homens. }\end{array}$ \\
\hline $\begin{array}{l}\text { Responsável financeiro } \\
\text { da família }\end{array}$ & 3,189 & 0,074 & $\begin{array}{l}\text { Com nível de significância de 10\%, indivíduos que são o } \\
\text { principal responsável financeiro da família tendem a ser } \\
\text { menos impulsivos nas compras. }\end{array}$ \\
\hline Uso do limite de cartão & 18,546 & 0,000 & $\begin{array}{l}\text { Indivíduos que utilizam um maior percentual do limite do } \\
\text { cartão de crédito tendem a ser mais impulsivos nas compras. }\end{array}$ \\
\hline Perfil de gastador & 15,165 & 0,004 & $\begin{array}{l}\text { Indivíduos que gastam mais do que seus rendimentos } \\
\text { suportam tendem a ser mais impulsivos nas compras. }\end{array}$ \\
\hline $\begin{array}{l}\text { Prazo para pagamento } \\
\text { (produtos de até R\$ } \\
500,00)\end{array}$ & 12,991 & 0,005 & $\begin{array}{l}\text { Indivíduos que optam por maior prazo para pagamento } \\
\text { em compras que custam até R } \$ 500,00 \text { tendem a ser mais } \\
\text { impulsivos. }\end{array}$ \\
\hline
\end{tabular}

Fonte: Elaborado pelos autores(2015). 
Tabela 3 - Análise de cruzamento entre variáveis categóricas

\begin{tabular}{|l|c|c|l|}
\hline Cruzamento & $\begin{array}{c}\text { Qui-qua- } \\
\text { drado }\end{array}$ & Valor-p & Conclusão \\
\hline $\begin{array}{l}\text { Perfil de poupador X Uso do } \\
\text { limite do cartão de crédito }\end{array}$ & 20,607 & 0,056 & $\begin{array}{l}\text { Indivíduos que poupam mais tendem a usar menos o limite } \\
\text { do cartão de crédito. }\end{array}$ \\
\hline $\begin{array}{l}\text { Perfil de gastador X Uso do } \\
\text { limite do cartão de crédito }\end{array}$ & 56,823 & 0,000 & $\begin{array}{l}\text { Indivíduos que gastam mais do que seus salários suportam } \\
\text { tendem a usar mais o limite do cartão de crédito. }\end{array}$ \\
\hline $\begin{array}{l}\text { Prazo para pagamento X } \\
\text { Uso do limite do cartão de } \\
\text { crédito }\end{array}$ & 37,189 & 0,000 & $\begin{array}{l}\text { Indivíduos que optam por maior prazo de parcelamento } \\
\text { tendem a usar mais o limite do cartão de crédito. }\end{array}$ \\
\hline $\begin{array}{l}\text { Perfil de poupador X } \\
\text { Responsável financeiro da } \\
\text { família }\end{array}$ & 8,254 & 0,083 & $\begin{array}{l}\text { Com nível de significância de 10\%, indivíduos que não são } \\
\text { o principal responsável financeiro da família tendem a pou- } \\
\text { par mais. }\end{array}$ \\
\hline $\begin{array}{l}\text { Prazo para pagamento X } \\
\text { Número de dependentes }\end{array}$ & 9,127 & 0,028 & $\begin{array}{l}\text { Indivíduos sem dependentes tendem a optar por menores } \\
\text { prazos de pagamento. }\end{array}$ \\
\hline
\end{tabular}

Fonte: Elaborado pelos autores(2015).

Sobre os produtos que mais geram impulsividade, os respondentes afirmaram que sentem maior dificuldade para controlar a compra não planejada quando adquirem, principalmente, roupas e calçados $(30,73 \%)$, alimentos $(27,24 \%)$ e produtos de beleza $(8,97 \%)$.

A figura 2 ilustra a associação entre o uso do limite do cartão de crédito e impulsividade nas compras dos indivíduos. O ponto em azul da categoria representa a média na variável "impulsividade nas compras". É possível concluir que o indivíduo que consome maior parte de seu limite do cartão de crédito tende a ter maior comportamento impulsivo nas compras. Essa conclusão pode ser confirmada por meio do teste Qui-quadrado (tabela 2).

A figura 3 confirma tendência de comportamento impulsivo dos clientes que costumam parcelar em mais vezes produtos que custam até $\mathrm{R} \$ 500$. Destaque à diferença entre consumidores que preferem pagar à vista e consumidores que preferem parcelar em mais de 6 vezes (vide complemento na tabela 2 para validar estas conclusões,).

Figura 2 - Relação positiva entre uso de limite do cartão e impulsividade nas compras

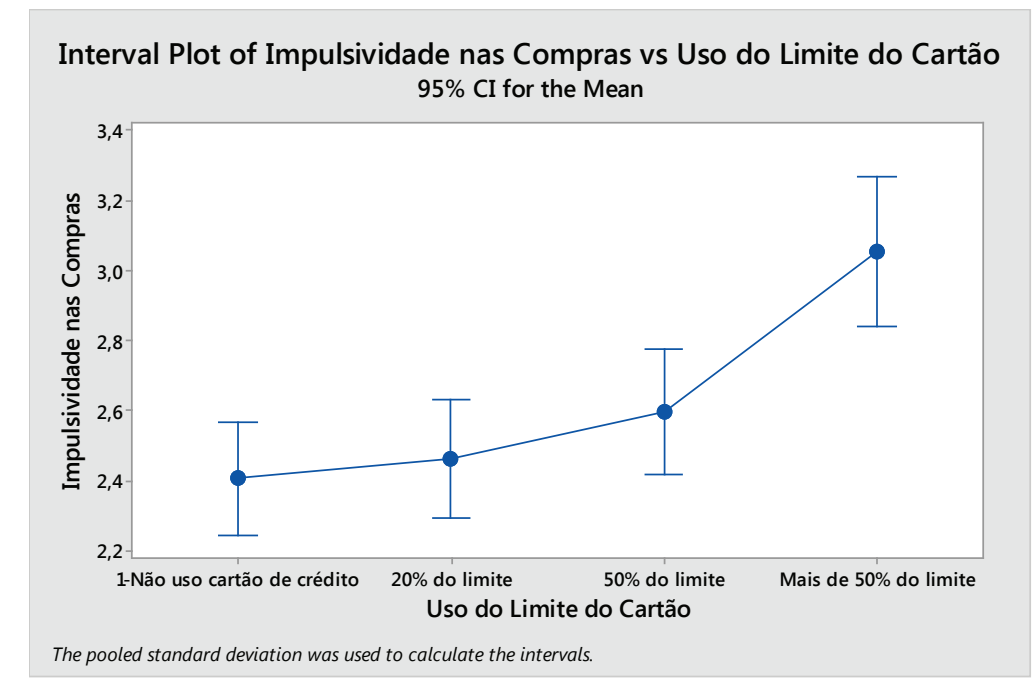

Fonte: Elaborado pelos autores(2015). 
Figura 3 - Relação positiva entre prazo para pagamento (produtos de até $R \$ 500$ ) e impulsividade nas compras

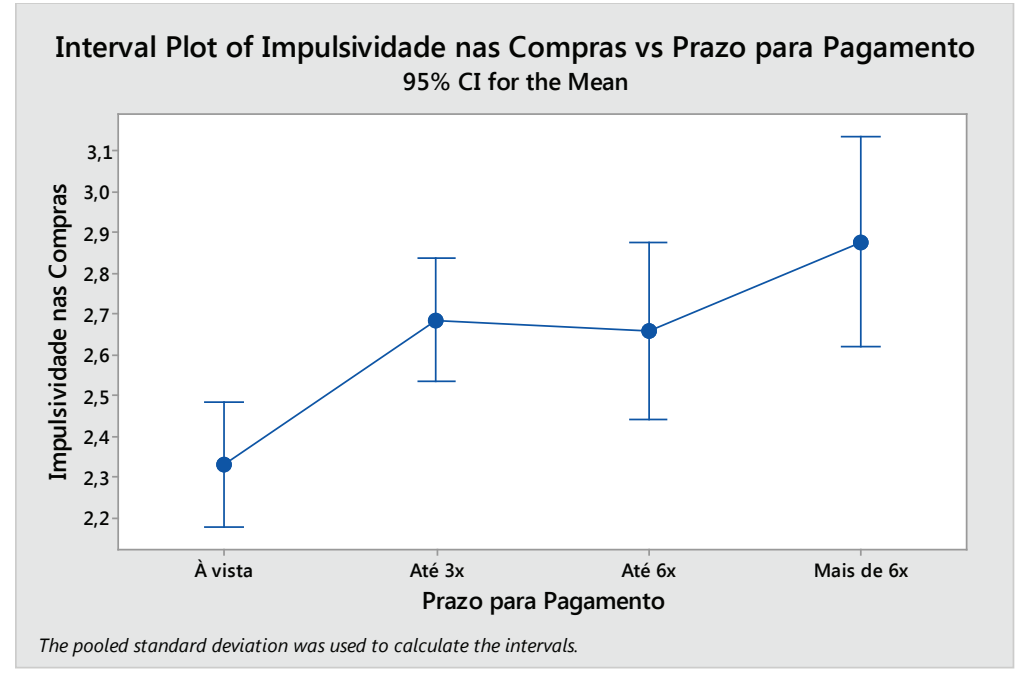

Fonte: Elaborado pelos autores(2015).

\subsection{Análise de equações estruturais}

\subsubsection{Avaliação do modelo de mensuração}

Antes de avaliar o modelo estrutural, procedeu-se com a verificação dos pressupostos de confiabilidade e validade (convergente e discriminante) dos modelos de mensuração.

A confiabilidade de uma escala está associada ao nível de confiança representado pelos indicadores dos construtos (HAIR et al., 2009). Este estudo seguiu as recomendações de Fornell e Lacker (1981), que indicam, no mínimo, 0,70 de confiabilidade composta como referência (MARÔCO, 2010). Outra análise relevante é a validade convergente, que mede o quanto os itens convergem para um mesmo conceito. Esse indicador tem como critério o valor da Variância Média Extraída (AverageVarianceExtrated) - AVE. Recomenda-se o índice mínimo de 0,50 em cada construto, indicando que pelo menos $50 \%$ da variância do construto foi incorporada na análise do modelo (CHIN, 2010).

A tabela 4 indica que todas as variáveis latentes apresentam valores AVE superiores a 0,50 , isto é, o equivalente a raiz de AVE de aproximadamente a 0,70, confirmando, assim, a validade convergente. Do mesmo modo, os índices de confiabilidade composta foram superiores a 0,70 em todos os construtos, indicando a confiabilidade da escala.

A validade discriminante permite verificar se os conceitos representados pelos construtos são suficientemente distintos (HAIR et al., 2009). Seguindo as recomendações de Fornell e Lacker (1981), para que uma variável latente seja distinta das demais, a sua raiz quadrada de AVE deve ser maior do que a correlação de Pearson com cada um dos demais construtos. Seguindo esse critério, a validade discriminante do modelo está atendida. Na tabela 4, os valores indicados ao longo da diagonal (em negrito) representam a raiz de AVE. A tabela apresenta a correlação entre as variáveis latentes propostas no modelo. As médias, desvios-padrão e coeficientes de variação foram calculados com os escores obtidos na estimação do modelo utilizando o software SmartPLS 2.0M3 (RINGLE; WENDE; WILL, 2005). 
Tabela 4 - Correlação de Pearson e estatísticas descritivas das variáveis latentes (VL).

\begin{tabular}{l|c|c|c|c|c}
\hline Variáveis & $\mathbf{1}$ & $\mathbf{2}$ & $\mathbf{3}$ & $\mathbf{4}$ & $\mathbf{5}$ \\
\hline 1-Ansiedade & 0,807 & & & & \\
2-Racionalidade & $-0,303$ & $\mathbf{0 , 8 0 8}$ & & & \\
3-Materialismo & 0,417 & $-0,378$ & $\mathbf{0 , 8 1 2}$ & & \\
4-Uso de Rec. Fin. & 0,260 & $-0,450$ & 0,433 & $\mathbf{0 , 8 3 6}$ & \\
5-Impulsividade & 0,426 & $-0,665$ & 0,364 & 0,486 & 0,814 \\
\hline AVE & 0,651 & 0,652 & 0,659 & 0,699 & 0,662 \\
\hline Confiab. Composta & 0,882 & 0,849 & 0,885 & 0,903 & 0,853 \\
R $^{2}$ & $17,3 \%$ & $26,1 \%$ & $\#$ & $18,8 \%$ & $52,8 \%$ \\
Média & 3,54 & 3,54 & 2,85 & 2,72 & 2,58 \\
Erro Padrão (MD) & 0,061 & 0,051 & 0,056 & 0,065 & 0,047 \\
Desvio Padrão & 0,956 & 0,793 & 0,880 & 1,015 & 0,738 \\
Coef. Variação & $27,01 \%$ & $22,40 \%$ & $30,87 \%$ & $37,33 \%$ & $28,59 \%$ \\
\hline
\end{tabular}

Fonte: Elaborado pelos autores(2015).

Outra forma de verificar a validade discriminante do modelo é por meio da análise das cargas cruzadas. Os resultados dessa análise mostram que os indicadores têm cargas mais altas em seus construtos e cargas mais baixas nos demais construtos, indicando validade discriminante. A significância foi estimada por meio de bootstrap com 244 casos e 1000 repetições, conforme tabela 5.

Tabela 5 - Cargas cruzadas para avaliação da validade discriminante

\begin{tabular}{|c|c|c|c|c|c|c|c|}
\hline Construto & Indicador & 1 & 2 & 3 & 4 & 5 & Valor-P \\
\hline \multirow{4}{*}{$\begin{array}{l}1 \\
\text { Ansiedade }\end{array}$} & $\begin{array}{l}\text { a. Quando compro um produto } \\
\text { que precisa ser entregue } \\
\text { em minha residência, fico } \\
\text { extremamente ansioso(a), } \\
\text { desejando que chegue o } \\
\text { quanto antes }\end{array}$ & 0,784 & $-0,221$ & 0,341 & 0,260 & 0,349 & 0,000 \\
\hline & $\begin{array}{l}\text { b. Quando quero alguma coisa, } \\
\text { não gosto de esperar }\end{array}$ & 0,802 & $-0,331$ & 0,355 & 0,243 & 0,392 & 0,000 \\
\hline & $\begin{array}{l}\text { c. Costumo "sofrer por } \\
\text { antecipação", ou seja, fico } \\
\text { preocupado antes mesmo de o } \\
\text { problema realmente surgir }\end{array}$ & 0,786 & $-0,155$ & 0,294 & 0,137 & 0,241 & 0,000 \\
\hline & d. Em geral, sou muito ansioso(a) & 0,853 & $-0,236$ & 0,340 & 0,179 & 0,361 & 0,000 \\
\hline \multirow{3}{*}{$\begin{array}{l}2 \\
\text { Racionalidade }\end{array}$} & $\begin{array}{l}\text { a. Geralmente eu compro coisas } \\
\text { sem pensar (escala reversa) }\end{array}$ & $-0,344$ & 0,847 & $-0,376$ & $-0,426$ & $-0,688$ & 0,000 \\
\hline & $\begin{array}{l}\text { b. Sempre planejo o meu } \\
\text { orçamento antes de comprar }\end{array}$ & $-0,110$ & 0,732 & $-0,231$ & $-0,303$ & $-0,383$ & 0,000 \\
\hline & $\begin{array}{l}\text { c. Sou muito mais emocional do } \\
\text { que racional durante as compras } \\
\text { (escala reversa) }\end{array}$ & $-0,222$ & 0,839 & $-0,279$ & $-0,336$ & $-0,465$ & 0,000 \\
\hline \multirow{4}{*}{$\begin{array}{l}3 \\
\text { Materialismo }\end{array}$} & $\begin{array}{l}\text { a. Eu seria mais feliz se tivesse } \\
\text { condições de comprar mais } \\
\text { coisas }\end{array}$ & 0,280 & $-0,269$ & 0,798 & 0,318 & 0,230 & 0,000 \\
\hline & $\begin{array}{l}\text { b. Fico muito chateado }(a) \\
\text { quando não consigo comprar as } \\
\text { coisas que gostaria }\end{array}$ & 0,437 & $-0,312$ & 0,850 & 0,373 & 0,276 & 0,000 \\
\hline & c. Ter bens materiais me faz feliz & 0,336 & $-0,347$ & 0,863 & 0,406 & 0,353 & 0,000 \\
\hline & $\begin{array}{l}\text { d. Eu gosto de muito luxo em } \\
\text { minha vida }\end{array}$ & 0,280 & $-0,296$ & 0,730 & 0,296 & 0,314 & 0,000 \\
\hline
\end{tabular}




\begin{tabular}{|c|c|c|c|c|c|c|c|}
\hline \multirow{4}{*}{$\begin{array}{l}4 \\
\text { Uso de Rec. } \\
\text { financeiros } \\
\text { disponíveis }\end{array}$} & $\begin{array}{l}\text { a. Costumo comprar mais } \\
\text { quando o pagamento pode ser } \\
\text { parcelado }\end{array}$ & 0,171 & $-0,344$ & 0,341 & 0,832 & 0,338 & 0,000 \\
\hline & $\begin{array}{l}\text { b. Quando tenho dinheiro ou } \\
\text { saldo no cartão de crédito } \\
\text { disponível para comprar, acabo } \\
\text { gastando }\end{array}$ & 0,287 & $-0,422$ & 0,405 & 0,846 & 0,466 & 0,000 \\
\hline & $\begin{array}{l}\text { c. Sempre tenho parcelas a } \\
\text { pagar }\end{array}$ & 0,207 & $-0,357$ & 0,319 & 0,819 & 0,383 & 0,000 \\
\hline & $\begin{array}{l}\text { d. Geralmente quando aumenta o } \\
\text { meu limite de crédito, eu compro } \\
\text { mais }\end{array}$ & 0,191 & $-0,374$ & 0,373 & 0,846 & 0,421 & 0,000 \\
\hline \multirow{3}{*}{$\begin{array}{l}5 \\
\text { Impulsividade }\end{array}$} & $\begin{array}{l}\text { a. Cenário de decisão (vide } \\
\text { Metodologia) }\end{array}$ & 0,256 & $-0,329$ & 0,213 & 0,341 & 0,674 & 0,000 \\
\hline & $\begin{array}{l}\text { b. Considerando que uma pessoa } \\
\text { impulsiva costuma comprar } \\
\text { espontaneamente, de forma } \\
\text { imediata e sem planejamento, } \\
\text { o quanto você se considera } \\
\text { impulsivo(a)? (Likertde5 pontos) }\end{array}$ & 0,386 & $-0,609$ & 0,351 & 0,394 & 0,870 & 0,000 \\
\hline & $\begin{array}{l}\text { c. Quando vou a uma LOJA } \\
\text { DE ROUPAS, em geral, me } \\
\text { enquadro na seguinte situação: } \\
\text { (Likert de } 5 \text { pontos) }\end{array}$ & 0,379 & $-0,626$ & 0,306 & 0,447 & 0,881 & 0,000 \\
\hline
\end{tabular}

Fonte: Elaborado pelos autores(2015).

\subsubsection{Avaliação do modelo estrutural}

O modelo representado na figura 4 foi estimado por meio do método PartialLeastSquares (PLS) e operacionalizado pelo software SmartPLS 2.0M3 (RINGLE; WENDE; WILL, 2005).

Figura 4 - Coeficientes do modelo estrutural e significâncias estimadas por Bootstrap.

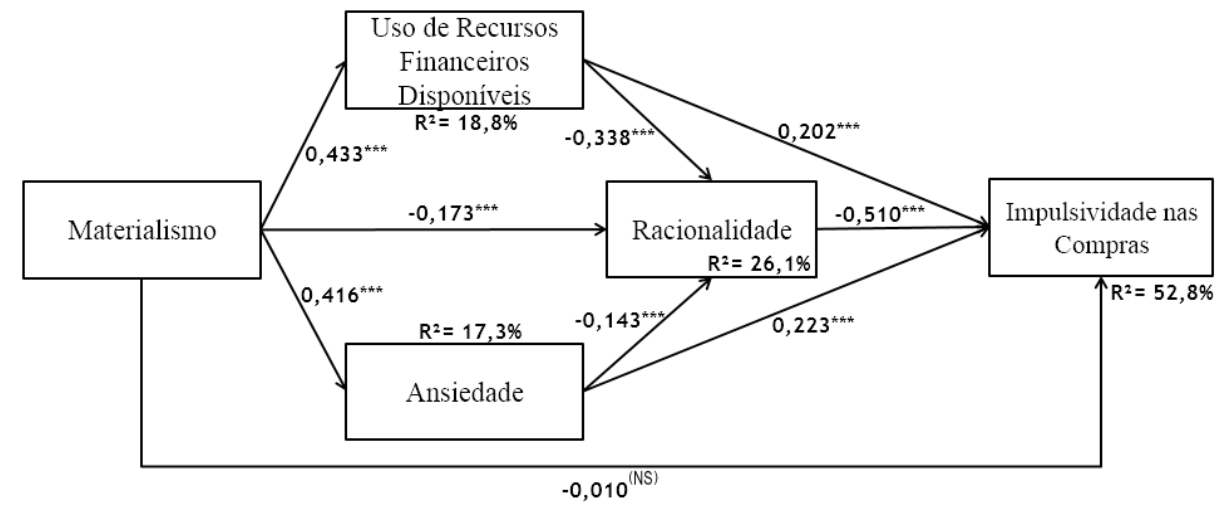

Fonte: Elaborado pelos autores(2015).

Nota: A significância foi estimada por bootstrap com N = 244 e 1000 repetições. Para não tornar a figura muito complexa, foram omitidos os indicadores, porém suas cargas fatoriais são apresentadas na tabela 5 . ${ }^{*}<<0,05 ;{ }^{* *} p<0,01$; ${ }^{* * *} p<0,001$; (NS) não significante

O modelo estrutural foi capaz de explicar $52,8 \%$ da variabilidade do construto dependente "impulsividade nas compras", objeto de estudo deste artigo. Entre coeficientes de caminho (beta) que apontam para esta variável, apresentam-se altamente significantes, com 0,202 ( $p<0,001)$ de "uso de recursos financeiros disponíveis", $0,223(p<0,001)$ de "ansiedade" e $-0,510(p<0,001)$ de "racionalidade", indicando que o aspecto racional $x$ emocional durante as compras pode explicar boa parte do comportamento 
impulsivo do indivíduo. O sinal negativo vindo de "racionalidade" $(-0,510)$ indica que, quanto mais racional for o indivíduo, menos impulsivo ele será. A mesma lógica ocorre com todas as outras relações em que o conceito racionalidade está envolvido.

A variável "materialismo" não se mostrou importante na explicação direta de "impulsividade nas compras" $(-0,010 ; p=0,74)$, mas é relevante o seu efeito total (tabela 6) presente no encadeamento de conceitos, considerando as variáveis mediadoras "uso de recursos financeiros disponíveis", "ansiedade" e "racionalidade".

Outro resultado que o modelo propõe está na capacidade do construto "materialismo" explicar parte da variabilidade de "ansiedade" $(0,416 ; p<0,001)$, "uso de recursos financeiros disponíveis" $(0,433 ; p<0,001)$ e "racionalidade" nas compras $(-0,173 ; p<0,001)$. Sendo assim, cabe ressaltar a importância de "ansiedade" e de "uso de recursos financeiros disponíveis" na explicação de "racionalidade", com betas iguais a 0,143 $(p<0,001)$ e $-0,338(p<0,001)$ respectivamente. A tabela 6 mostra os efeitos totais, considerando as relações diretas e indiretas do modelo.

Tabela 6 - Efeito total (efeito direto + indireto), considerando variáveis mediadoras

\begin{tabular}{l|c|c}
\hline Construtos & Racionalidade & Impulsividade \\
\hline Ansiedade & - & 0,2926 \\
\hline Materialismo & $-0,378$ & $\mathbf{0 , 3 7 0 5}$ \\
\hline Financeiro & - & 0,3717 \\
\hline
\end{tabular}

Fonte: Elaborado pelos autores(2015).

O efeito direto de "materialismo" sobre a "impulsividade" nas compras não apresentou significância estatística. Ao analisar o efeito total considerando as variáveis mediadoras, entretanto, o resultado foi $0,3705$ ( $p<0,001)$, confirmando que o fato de o indivíduo ser materialista não garante sua impulsividade nas compras. Para tanto, seria necessária a interação com as variáveis mediadoras "ansiedade", "uso de recursos financeiros disponíveis" e "racionalidade".

\subsubsection{Efeito moderador nos coeficientes estruturais}

Para verificar se alguma variável atua como moderadora nas relações que apontam para o construto dependente "impulsividade nas compras", efeitos interativos foram aplicados entre cada uma das variáveis independentes e a variável moderadora. Para averiguar possíveis efeitos moderadores das variáveis categóricas, uma nova variável foi incluída no modelo e vinculada ao construto dependente "impulsividade nas compras". Os produtos resultantes da multiplicação entre os indicadores da VI (variável independente) e as categorias da variável moderadorasão os indicadores dessa nova variável. Os valores dos indicadores foram padronizados antes da multiplicação. O procedimento foi realizado para cada uma das variáveis categóricas moderadoras das relações que envolvem o construto "impulsividade nas compras". Os resultados podem ser consultados na tabela 7 . 
Tabela 7 - Efeito moderador

\begin{tabular}{llccc}
\hline Relação & Variável moderadora & $\begin{array}{c}\text { Beta (Var. } \\
\text { Mod. sobre VL } \\
\text { Impulsividade) }\end{array}$ & $\begin{array}{c}\text { Efeito } \\
\text { moderador }\end{array}$ & $\begin{array}{c}\text { Valor-P } \\
\text { (Moderação) }\end{array}$ \\
\hline $\begin{array}{l}\text { Uso recursos financeiros } \\
\text { disponíveis } \rightarrow \\
\text { impulsividade }\end{array}$ & $\begin{array}{l}\text { Sexo (se masculino) } \\
\text { Responsável Financeiro } \\
\text { Uso do Limite de Cartão de } \\
\text { Crédito }\end{array}$ & $-0,102^{* * *}$ & $-0,115^{* * *}$ & 0,0000 \\
$\begin{array}{l}\text { Materialismo } \rightarrow \\
\text { Impulsividade }\end{array}$ & Responsável Financeiro & $-0,131^{* * *}$ & $0,116^{* *}$ & 0,0089 \\
$\begin{array}{l}\text { Racionalidade } \rightarrow \\
\text { Impulsividade }\end{array}$ & Número de Dependentes & $-0,092^{* * *}$ & $-0,134^{* * *}$ & 0,0000 \\
$\begin{array}{l}\text { Ansiedade } \rightarrow \\
\text { Impulsividade }\end{array}$ & Uso do Limite de Cartão de & $-0,088^{* * *}$ & $0,067^{* *}$ & 0,0088 \\
\hline
\end{tabular}

Fonte: Elaborado pelos autores(2015).

Nota:Foram incluídas apenas as variáveis moderadoras significantes.

A tabela 7 indica a presença de efeito moderador em algumas relações de dependência, gerado pela ação de variáveis de controle. O efeito da variável "Uso de recursos financeiros disponíveis" sobre "Impulsividade" sofre redução quando consideradas as variáveis "Sexo" (se masculino) e quando o indivíduo é o principal responsável financeiro da família, mas sofre aumento quando considerados apenas os indivíduos que usam mais o limite do cartão de crédito. As relações entre "Materialismo" e "Impulsividade", bem como "Ansiedade" e "Impulsividade" sofrem redução em seus coeficientes estruturais quando consideradas as variáveis "Responsável financeiro" e "Uso do limite de cartão de crédito", respectivamente. Por fim, o efeito de racionalidade sobre impulsividade sofre aumento ao avaliar a moderação da variável "Número de dependentes", ou seja, quando o indivíduo possui dependentes financeiros.

Os achados que mostram alguns efeitos de moderação no modelo não afetam a qualidade da estrutura apresentada na figura 2, uma vez que, mesmo avaliando tais efeitos, as conclusões lógicas das relações propostas na estrutura continuam semelhantes, com pequenas mudanças para mais ou para menos nos coeficientes beta.

\section{Discussão dos Resultados}

A relação positiva entre "materialismo" e o "uso dos recursos financeiros" (H1) pode ser explicada pelo forte sentimento que pessoas materialistas têm de que sua felicidade e sucesso estão atrelados à posse de bens luxuosos (RICHINS; DAWSON,1992). Outro indício é o fato de que pessoas materialistas se comparam com pessoas de maior poder aquisitivo e buscam se igualar a elas, o que as leva a gastar excessivamente.

O estudo revelou uma relação negativa entre "materialismo" e "racionalidade" (H2). Os materialistas, de modo geral, tendem a atribuir maior grau de emotividade às compras, e não as planejam racionalmente. Isto é, privilegiam os sentimentos e emoções antes da tomada de decisão (ASSMAR; RODRIGUES, 1989), normalmente vinculada aos fatores situacionais (AMOS et al., 2014).

A relação entre "materialismo" e "ansiedade" (H3) mostrou-se forte neste estudo, mostrando que o materialismo pode gerar ansiedade pela compra de novos itens (RICHINS; DAWSON,1992). Para o gestor, além de identificar o perfil materialista do consumidor, deve-se avaliar de que forma o indivíduo (ou a maioria do seu público-alvo) se comporta em relação ao seu nível de ansiedade, racionalidade nas compras e uso de recursos financeiros disponíveis. Com isso, é possível avaliar se o cliente materialista será mais impactado por apelos emocionais ou racionais das campanhas de marketing.

O modelo de equações estruturais não confirmou a influência direta do materialismo sobre a impulsividade nas compras. Um indivíduo materialista, não ansioso, que saiba usar os recursos financeiros 
disponíveis e que não seja emotivo durante as compras, provavelmente não terá comportamento impulsivo. O caráter materialista, por si só, não é determinante da compra não planejada (H4), contrariando em parte Podoshen e Andrzejewski (2012) e Badgaiyan e Yerma, (2014). Porém as evidências indicaram efeito indireto desse construto sobre o comportamento impulsivo do cliente. Se um indivíduo for ansioso, usar seus recursos financeiros e se caracterizar mais pela emotividade que pela racionalidade, tenderá a ser impulsivo. A relação positiva entre o "uso dos recursos financeiros" e a "impulsividade" (H6) foi encontrada por outros pesquisadores (AWAN; ABBAS, 2015; BASHAR; AHAMAD; WASIG, 2013; BEATTY; FERRELL, 1998), e sua relação negativa com "racionalidade" (H5) mostra que os consumidores que utilizam maior percentual do limite do cartão de crédito e que gastam mais do que ganham são mais impulsivos nas compras, ou seja, são mais emotivos e mais suscetíveis aos estímulos que levam ao consumo, conclusões similares a Amos et al. (2014).

A relação negativa entre "ansiedade" e "racionalidade" $(\mathrm{H} 7)$ foi comprovada neste estudo. Esse fenômeno pode ser explicado pelo perfil mais emotivo dos indivíduos ansiosos, que acabam comprando impulsivamente para satisfazer suas necessidades imediatas (ARNOLD; PRICE; ZINKHAN, 2004).

A compra impulsiva está ligada, além de outros fatores, ao estado emocional do indivíduo. Dessa forma, a relação positiva entre "ansiedade" e "impulsividade" $(\mathrm{H} 8)$ pode ser explicada pela necessidade das pessoas ansiosas aplacarem seu estado emocional negativo (VLACHOS, 2010) por meio das compras, seja pela gratificação imediata deste processo,seja pela vontade repentina de comprar algo imediatamente.

Para os gestores de marketing, esses resultados podem ser úteis para a elaboração de abordagens que atinjam de maneira mais efetiva cada grupo de consumidores. Destaca-se sua utilidade nas abordagens one-to-one, considerando as características predominantes do perfil do público-alvo, especialmente quanto ao seu grau de "ansiedade", "uso de recursos financeiros disponíveis" e "racionalidade".No caso de marketing em massa, pode-se customizar a abordagem ou priorizar recursos publicitários visuais e linguísticos de acordo com o perfil de cliente almejado (CARDOSO, 2004; SHAW, 2001).

\section{Conclusão}

Neste estudo, foi possível avaliar a relação entre materialismo e comportamento de compra impulsivo quando mediada pelos construtos de racionalidade, uso de recursos financeiros, nível de ansiedade e racionalidade. Pode-se concluir, a partir dos resultados, que: se o indivíduo for ansioso, usar seus recursos financeiros disponíveis e for mais emocional que racional, tenderá a ser impulsivo.

Mapear o comportamento do consumidor quanto ao seu perfil racional ou emotivo durante as compras, e ainda quanto às suas características demográficas e de padrão de consumo, tais como a preferência de parcelamento, pode trazer vantagens à organização, pois ela poderá dispor de mais informações para customizar a abordagem de marketing e a oferta de produtos e serviços apropriados.

Como limitação do estudo, destaca-se a utilização de amostra não probabilística por conveniência (e-mail e redes sociais) e projeção dos resultados voltada apenas para a grande São Paulo.

Estudos futuros podem revelar novas descobertas acerca do papel do materialismo sobre o comportamento impulsivo nas compras, assim como testar na prática o impacto dos apelos publicitários sobre a decisão de compra para cada perfil de consumidor (emotivo ou racional).

\section{Referências}

AMOS, C.; HOLMES, G. R.; KENESON, W. C. A meta-analysis of consumer impulse buying.Journal of Retailing and Consumer Services, [S.I], v. 21, n. 2, p. 86-97, Mar. 2014.

ARNOLD, E.; PRICE, L.; ZINKHAN, G..Consumers. New York: McGraw-Hill, 2004.

ASSMAR, E. M. L.; RODRIGUES, A. Uma escala para medir racionalidade/emocionalidade. Arquivos Brasileiros de Psicologia, Rio de Janeiro, v. 41, n. 2, p. 22-28, $2^{\circ}$ trim.-maio 1989.

AWAN, A. G.; ABBAS, N. Impact of demographic factors on impulse buying behavior of consumers in 
multan-pakistan. European Journal of Business and Management,[S.I], v.7,n..22, 2015.

BADGAIYAN, A. J.; VERMA, A.. Intrinsic factors affecting impulsive buying behavior: evidence from India. Journal of Retailing and Consumer Services, [S.I], v. 21, n. 4, p. 537-549, Jul. 2014.

BASHAR, A.; AHMAD, I.; WASIQ, M. A study of influence of demographic factors on consumer impulse buying behavior. Journal of Management Research, [S.I],v. 13, n. 3, p. 145-154, 2013.

BEATTY, S.; FERRELL, M. E. Impulse buying: modeling its precursors. Journal of Retailing, [S.I], v. 74, n. 2, p. $169-191$, Summer 1998.

BLACKWELL, R. D.; MINIARD, P. W.; ENGEL, J. F. Comportamento do consumidor. 8ed. Rio de Janeiro: Livros Técnicos e Científicos, 2008.

CARDOSO, P. R. Os apelos racionais e emocionais na publicidade: uma análise conceptual. Covilhã/ Portugal: LabCom, 2005.

CHIN, W. W.; NEWSTED, P. R. Structural equation modeling analysis with small samples using partial least squares. In: RICK H. HOYLE (Ed.). Statistical strategies for small sample research. Thousand Oaks: Sage, 1999. p.307-341.

COHEN, J. Statistical Power Analysis for the Behavioral Sciences. $2^{a}$.ed. New York: Psychology Press, 1988.

COSTA, F. C. X.; LARÁN, J. A. Influências do ambiente de loja e o comportamento de compra por impulso: a visão dos clientes de lojas virtuais. Revista de Administração USP, São Paulo, v.41, n.1, p. 96-106, jan./fev./mar.2006.

CRASKE, M. G. et al. What is an anxiety disorder?. Depression and anxiety, [S.I], v. 26, n. 12, p. 10661085, 2009.

CUNHA, R. A. N. Comportamento de compra por impulso: proposta de um modelo de mensuração relacional. 2012. 220 f. Tese (Doutorado em Administração) - Universidade Nove de Julho, São Paulo, 2012.

DIAS, S. E. F. et al. Efeitos das estratégias de marketing de compras coletivas sobre o comportamento impulsivo. Revista Brasileira de Marketing, São Paulo, v. 13, n. 3, p. 138-151, abril/jun. 2014.

DITTMAR, H. Compulsive buying: a growing concern? An examination of gender, age, and endorsement of materialistic values as predictors. British Journal of Psychology, [S.I],v.96, n.4, 467-491, Nov. 2005.

DONTHU, N.; GILLILAND, D. Observations: the infomercial shopper. Journal of Advertising Research, New York-NY, v. 36, n. 2, p. 69-76, 1996.

FAUL, F. et al. Statistical power analysis using $G^{*}$ Power 3.1: Tests for correlation and regression analyses. Behavior research methods, [S.I], v. 41, n. 4, p. 1149-1160, Nov. 2009.

FELIPE, I. J. D. S.; CERIBELI, H. B.; BRANDÃO, R. M. Antecedentes da Compra Compulsiva dos Universitários em Mariana (MG). RevistaCiênciasAdministrativas, Fortaleza, v. 23, n. 2, p. 322-335, maio/ago.2017.

FORNELL, C.; LARCKER, D. F. Structural equation models with unobservable variables and measurement error: Algebra and statistics. Journal of marketing research, [S.I], v. 18, n. 3, p. 382-388, Aug.1981.

FRALEY, R. C.; WALLER, N. G.; BRENNAN, K. A. An item response theory analysis of self-report measures of adult attachment. Journal of personality and social psychology, Washington, v. 78, n. 2, p. 350-365, 2000.

GOLDSMITH, R. E.; CLARCK, R. A. Materialism, Status Consumption, and Consumer Independence. The Journal of Social Psychology, London, v.152, n.1, p.43-60, 2012. 
HAIR Jr., J. F. et al.Multivariate Data Analysis. 7th ed. New Jersey: Prentice Hall, 2009.

HAIR, J. et al. A primer on partial least square structural equation modeling (plssem). Thousand Oaks: Sage, 2014.

MARÔCO, J. Análise de equações estruturais: fundamentos teóricos, software \& aplicações. ReportNumber, Lda: Pedro Pinheiro, 2010.

MICK, D. G. Are studies of dark side variables confounded by socially desirable responding? The case of materialism. Journal of consumer research, [S.I], v.23, n.2, p. 106-119, 1996.

MISHRA, H. G. et al. Buying Impulsive Trait: An effective moderator for shopping emotions and perceived risk. Indian Institute of Management, [S.I], v.14, n.2, p.109, 2014.

PIRON, F. Defining impulse purchasing. In: HOLMAN, R.H.; SOLOMON, Michael R. NA-Advances in ConsumerResearch, Provo, UT : Association for Consumer Research, 1991. v.. 18, p. 509-514.

PODOSHEN, J. S.; ANDRZEJEWSKI, S. A. An examination of the relationships between materialism, conspicuous consumption, impulse buying, and brand loyalty. Journalof Marketing TheoryandPractice, [S.I], v. 20, n. 3, p. 319-334, 2012.

PORPINO, G.; PARENTE,J. Antecedentes e consequências da compra impulsiva: um estudo exploratório. In: CONGRESSO ONLINE - ADMINISTRAÇÃO, 11., 2014, São Paulo. Anais... São Paulo: CONVIBRA, 2014. p. 1-17. Disponível em: <http://www.convibra.com.br/upload/ paper/2013/37/2013_37_8213.pdf>. Acessoem: 01 jun. 2016.

PURI, R. Measuring and modifying consumer impulsiveness: A cost-benefit accessibility framework. Journal of Consumer Psychology, [S.I], v. 5, n. 2, p. 87-113, 1996.

RICHINS, M. L.; DAWSON, S. A consumervaluesorientation for materialismand its measurement: Scaledevelopmentandvalidation. Journal of consumerresearch, [S.I], v. 19, n. 3, p. 303-316, 1992.

ROOK, D. W. The buying impulse. Journal of consumerresearch, [S.I], v. 14, n. 2, p. 189-199, 1987.

ROOK, D. W.; FISHER, R. J.

Normativeinfluencesonimpulsivebuyingbehavior. Journalofconsumerresearch, $[S, I]$, v. 22, n. 3, p. 305313, 1995.

STEFANINI, C. J.; OLIVEIRA, B. Fatores relacionados à compra compulsiva. In: ENCONTRO DA ANPAD (EnANPAD), 38., 2014, Rio de Janeiro. Anais... Rio de Janeiro: ANPAD, 2014. p. 1-14.

STERN, H. The significance of impulse buyingtoday. Journal of Marketing, [S.I], v. 26, n. 2, p. 59-62, Abr. 1962.

SHAW, M. J. et al. Knowledge management and data mining for marketing. Decisionsupport systems, [S.I], v. 31, n. 1, p. 127-137, May. 2001.

TEIXEIRA, J. M.; HERNANDEZ, J.M. C. Valores de compra hedônico e utilitário: os antecedentes e as relações com os resultados do varejo. Revista Eletrônica de Administração, Porto Alegre, v. 71, n. 1, p. 130-160, jan./abr. 2012.

VELUDO-DE-OLIVEIRA, T. M.; IKEDA. A. A.; SANTOS, R. C. Compra Compulsiva e a Influência do Cartão de Crédito. Revista de Administração de Empresas. FGV EAESP, v. 44, n. 3, p. 89-99, jul./set. 2004.

VLACHOS, P. A. et al. Consumer-retailer emotional attachment: Some antecedents and the moderating role of attachment anxiety. European Journal of Marketing, [S.I], v. 44, n. 9/10, p. 1478-1499, 2010.

WEUN, S.; JONES, M. A.; BEATTY, S. E. A parsimonious scale to measure impulse buying tendency. In: AMA Educators' Proceedings, 8., 1997, Chicago. Anais... Chicago: American Marketing Association: Enhancing knowledge development in marketing: 1997. p. 8-26. 
YOUN, S; FABER, R. Impulse buying: its relation to personality traits and cues. In: $\mathrm{HOCH}$, S. J.;

MEYER, R. J. Na-Advances in Consumer Research. [S.I]: Association for Consumer Research, 2000. v. 27. p. $179-185$

XIAO, S. H.; NICHOLSON, M.. A multidisciplinary cognitive behavioural framework of impulse buying: a systematic review of the literature. International Journal of Management Reviews, [S.I], v. 15, n. 3, p. 333-356, 2013.

YANG, K.; FORNEY, J. C. The moderating role of consumer technology anxiety in mobile shopping adoption: Differential effects of facilitating conditions and social influences. Journal of Electronic Commerce Research, [S.I], v. 14, n. 4, p. 334, 2013.

Submetido em: $12 / 12 / 2016$

Aprovado em: 28/07/2017 